\title{
A NOTE ON THE HARDY-HILLE AND MEHLER FORMULAS
}

\author{
$b y$ W. A. AL-SALAM and L. CARLITZ $\dagger$
}

(Received 21 September, 1964)

1. Let $L_{n}^{(\alpha)}(x)$ and $H_{n}(x)$ be the $n$th Laguerre and Hermite polynomials, respectively. Two well-known bilinear generating formulas are the Hardy-Hille formula [1, p. 101]

$$
\sum_{n=0}^{\infty} \frac{n !}{(1+\alpha)_{n}} L_{n}^{(\alpha)}(x) L_{n}^{(\alpha)}(y) t^{n}=(1-t)^{-\alpha-1} e^{-t(x+y) /(1-t)} F_{1}\left(-; 1+\alpha ; \frac{x y t}{(1-t)^{2}}\right)
$$

and the Mehler formula [1, p. 377]

$$
\sum_{n=0}^{\infty} H_{n}(x) H_{n}(y) \frac{t^{n}}{n !}=\left(1-4 t^{2}\right)^{-\frac{1}{t}} \exp \left\{-\frac{4 t^{2}\left(x^{2}+y^{2}\right)}{1-4 t^{2}}+\frac{4 x y t}{1-4 t^{2}}\right\} .
$$

This suggests the following problem. Consider the equation

$$
\sum_{n=0}^{\infty} \gamma_{n} f_{n}(x) f_{n}(y) t^{n}=f(t) e^{\left(x^{k}+y^{k}\right) a(t)} g\{x y c(t)\},
$$

where $f_{n}(x)$ is a polynomial in $x$ of degree $n$ with highest coefficient equal to 1 ,

$$
\begin{aligned}
& a(t)=\sum_{n=k}^{\infty} a_{n} t^{n}, \quad c(t)=\sum_{n=1}^{\infty} c_{n} t^{n}, \\
& f(t)=\sum_{n=0}^{\infty} A_{n} t^{n}, \quad g(t)=\sum_{0}^{\infty} B_{n} t^{n},
\end{aligned}
$$

$A_{0}=B_{0}=1$. We shall also assume that $a_{k}=1$ and $\gamma_{0} \gamma_{1} \gamma_{2} \ldots \gamma_{k-1} \neq 0$. We seek all sets of polynomials $\left\{f_{n}(x)\right\}$ which satisfy (1.3), (1.4) and (1.5).

We shall prove the following

THEOREM. The only solution of the functional equation (1.3), such that (1.4) and (1.5) hold, is given by

and

$$
\begin{aligned}
f_{s+n k}(x) & =n ! A^{n} x^{s} L_{n}^{(\alpha+2 s / k)}\left(x^{k} / A\right) \quad(s=0,1, \ldots, k-1), \\
f(t) & =\left(1+A t^{k}\right)^{-\alpha-1} \\
c(t) & =c_{1} t\left(1+A t^{k}\right)^{-2 / k} \\
a(t) & =\frac{t^{k}}{1+A t^{k}}
\end{aligned}
$$

where $\alpha, A$ are arbitrary constants.

$$
g(t)=\sum_{s=0}^{k-1} \frac{\gamma_{s} t^{s}}{c_{1}^{s}} F_{1}\left(-; \alpha+1+2 s / k ;-\frac{t^{k}}{A c_{1}^{k}}\right)
$$

† Supported in part by National Science Foundation grant GP-1593. 
2. Proof of the theorem. If we replace $y$ by $1 / y$ and $t$ by $t y$ in (1.3) and then put $y=0$, we get

$$
\sum_{0}^{\infty} \gamma_{n} f_{n}(x) t^{n}=e^{t k} g\left(x t c_{1}\right)
$$

This in the same way leads to

$$
\sum_{0}^{\infty} \gamma_{n} t^{n}=g\left(c_{1} t\right)
$$

Formulas (2.1) and (2.2) give

$$
\gamma_{n} f_{n}(x)=\sum_{r} \frac{\gamma_{n-k r}}{r !} x^{n-k r}
$$

By differentiating (2.3) $s$ times $(0 \leqq s<k)$, we see that

$$
\gamma_{n k+s} f_{n k+s}^{(s)}(0)=\frac{s ! \gamma_{s}}{n !} \quad(0 \leqq s<k)
$$

so that $\gamma_{n k+s} \neq 0$ for $s=0,1, \ldots, k-1$. This obviously implies that $\gamma_{n} \neq 0$ for all $n$.

Putting $y=0$ in (1.3) we get

$$
\sum_{n=0}^{\infty} f_{k n}(x) \frac{t^{n k}}{n !}=f(t) e^{x^{k} a(t)}
$$

which yields, on putting $x=0$,

$$
\sum_{n=0}^{\infty} \frac{t^{k n}}{\gamma_{k n}(n !)^{2}}=f(t)
$$

From (2.6) we get

$$
t f^{\prime}(t)=k \sum_{n=0}^{\infty} \frac{t^{k(n+1)}}{n !(n+1) ! \gamma_{k(n+1)}} .
$$

On the other hand, if we differentiate $(2.5) k$ times with respect to $x$, we get

$$
\sum_{n=0}^{\infty} f_{k n}^{(k)}(0) \frac{t^{k n}}{n !}=k ! a(t) f(t)
$$

But we have from (2.3)

$$
f_{k n}^{(k)}(0)=\frac{k ! \gamma_{k}}{(n-1) ! \gamma_{k n}} \quad(n \geqq 1),
$$

so that $(2.8)$ becomes

$$
a(t) f(t)=\gamma_{k} \sum_{n=0}^{\infty} \frac{t^{k(n+1)}}{n !(n+1) ! \gamma_{k(n+1)}}
$$


Comparing (2.9) with (2.7) we get

$$
\gamma_{k} t f^{\prime}(t)=k a(t) f(t)
$$

In the same way, we obtain from (2.5),

$$
\sum_{0}^{\infty} f_{k n}^{(2 k)}(0) \frac{t^{k n}}{n !}=\frac{(2 k) !}{2}(a(t))^{2} f(t)
$$

which is rewritten as

$$
\{a(t)\}^{2} f(t)=2 \gamma_{2 k} \sum_{n=0}^{\infty} \frac{t^{k(n+2)}}{n !(n+2) ! \gamma_{k(n+2)}} .
$$

On the other hand, we see from (2.6) that

$$
t^{k+1}\left\{t^{1-k} f^{\prime}(t)\right\}^{\prime}=k^{2} \sum_{0}^{\infty} \frac{t^{n(k+2)}}{n !(n+1) ! \gamma_{k(n+2)}} .
$$

Now (2.12) and (2.13) give

$$
\gamma_{k}^{2} t\left\{f^{\prime}(t)\right\}^{2}=2 \gamma_{2 k}\left\{t f^{\prime \prime}(t)-(k-1) f^{\prime}(t)\right\} f(t) .
$$

Hence

$$
f(t)=\left(1+A t^{k}\right)^{-\alpha-1}
$$

where $A$ and $\alpha$ are constants.

From (2.15) and (2.10) we get

$$
a(t)=\frac{t^{k}}{1+A t^{k}}
$$

Let us next differentiate (1.3) with respect to $y$ and put $y=0$. We get

$$
c_{1} \sum_{0}^{\infty} f_{n k+1}(x) \frac{t^{n k+1}}{n !}=x f(t) c(t) e^{x^{k} a(t)}
$$

Now if we differentiate (2.17) once with respect to $x$ and put $x=0$, we obtain

$$
f(t) c(t)=\gamma_{1} c_{1} \sum_{n=0}^{\infty} \frac{t^{n k+1}}{(n !)^{2} \gamma_{n k+1}}
$$

on the other hand, if we differentiate (2.17) $k+1$ times with respect to $x$ and put $x=0$, we get

$$
f(t) c(t) a(t)=c_{1} \gamma_{k+1} \sum_{n=0}^{\infty} \frac{t^{1+k(n+1)}}{n !(n+1) ! \gamma_{1+k(n+1)}}
$$

Comparing (2.19) and (2.18) we get

$$
t^{2}\left\{\frac{f(t) c(t)}{t}\right\}^{\prime}=\frac{k \gamma_{1}}{\gamma_{k+1}} f(t) c(t) a(t)
$$


Hence

$$
c(t)=c_{1} t\left(1+A t^{k}\right)^{\mu}
$$

where $\mu$ is a constant.

If we now differentiate (2.9) with respect to $t$, we get

$$
t\left\{a^{\prime}(t) f(t)+a(t) f^{\prime}(t)\right\}=k \gamma_{k} \sum_{0}^{\infty} \frac{t^{k(n+1)}}{(n !)^{2} \gamma_{k(n+1)}} .
$$

If we take the $k$ th derivative with respect to $x$ and with respect to $y$ and then put $x=y=0$, we get

$$
c_{1}^{k} \gamma_{k} \sum_{n=0}^{\infty} \frac{t^{k(n+1)}}{\gamma_{k(n+1)}(n !)^{2}}=f(t)\{c(t)\}^{k}+\frac{c_{1}^{k}}{\gamma_{k}}\{a(t)\}^{2} f(t) .
$$

Comparing this formula with (2.21), we obtain

$$
\gamma_{k} c_{1}^{k} t\left\{a^{\prime}(t) f(t)+a(t) f^{\prime}(t)\right\}=k \gamma_{k} f(t)\{c(t)\}^{k}+k c_{1}^{k}\{a(t)\}^{2} f(t) .
$$

This, together with (2.10), yields

$$
c_{1}^{k} t^{\prime}(t)=k\{c(t)\}^{k}
$$

Formulas (2.22), (2.20) and (2.16) require that

$$
\mu k=-2 .
$$

To determine $g(t)$ we differentiate (1.3) $s$ times with respect to $y$ and put $y=0$ to get

$$
c_{1}^{s} \sum_{0}^{\infty} f_{s+k n}(x) \frac{t^{s+k n}}{n !}=x^{s} f(t)\{c(t)\}^{s} e^{x^{k} a(t)} \quad(0 \leqq s<k),
$$

which itself leads to

$$
\gamma_{s} c_{1}^{s} \sum_{0}^{\infty} \frac{t^{s+k n}}{\gamma_{s+k n}(n !)^{2}}=f(t)\{c(t)\}^{s} .
$$

Comparing coefficients of $t^{s+k n}$ we get

$$
\gamma_{s+k n}=\frac{(-1)^{n} A^{-n} \gamma_{s}}{(\alpha+1+2 s / k)_{n}} \quad(0 \leqq s<k) .
$$

Consequently we obtain from (2.2) and (2.26)

$$
g(t)=\sum_{s=0}^{k-1} \frac{\gamma_{s}}{c_{1}^{s}} t_{0}^{s} F_{1}\left(-; \alpha+1+2 s / k ; \frac{t^{k}}{c_{1}^{k} A}\right)
$$

Putting (2.26), (2.24), (2.20), (2.16), (2.15) in (1.3) we get

$\sum \gamma_{n} f_{n}(x) f_{n}(y) t^{n}$

$=\left(1+A t^{k}\right)^{-\alpha-1} \exp \left\{\frac{\left(x^{k}+y^{k}\right) t^{k}}{1+A t^{k}}\right\}_{s=0}^{k-1} \frac{\gamma_{s} x^{s} y^{s} t^{s}}{\left(1+A t^{k}\right)^{2 s / k}}{ }_{0} F_{1}\left(-; \alpha+1+2 s / k ;-\frac{x^{k} y^{k} t^{k}}{A\left(1+A t^{k}\right)^{2}}\right)$. 
Comparing (2.27) with (1.1) we see that

$$
f_{s+k n}(x)=n ! A^{n} x^{s} L_{n}^{(\alpha+2 s / k)}\left(x^{k} / A\right) \quad(0 \leqq s \leqq k-1) .
$$

Note that $\gamma_{0}=1, \gamma_{1}, \gamma_{2}, \ldots, \gamma_{k-1}$ are arbitrary.

This completes the proof of the theorem.

If $k=1$ we see that the only solution of the functional equation

$$
\sum_{n=0}^{\infty} \gamma_{n} f_{n}(x) f_{n}(y) t^{n}=f(t) e^{(x+y) a(t)} g\{x y c(t)\}
$$

where $f(t), a(t), g(t), c(t)$ are defined as before, is essentially (1.1).

In case $k=2$ we see that the solution of the functional equation

$$
\sum_{n=0}^{\infty} \gamma_{n} f_{n}(x) f_{n}(y) t^{n}=f(t) e^{\left(x^{2}+y^{2}\right) a(t)} g\{x y c(t)\}
$$

is obtained from (1.1) in the following manner:

Denote the right-hand member of $(1.1)$ by $F_{\alpha}(x, y, t)$. We then exhibit the general solution of (2.29) by replacing the right member by

$$
F_{\alpha}\left(x^{2} y^{2} t^{2}\right)+B x y t F_{\alpha+1}\left(x^{2} y^{2} t^{2}\right),
$$

where $B$ is a non-zero constant.

The special case $\alpha=-\frac{1}{2}$ leads to the Mehler formula (1.2). However it is not necessary to assume $\alpha=-\frac{1}{2}$.

We remark that

and

$$
f_{2 n}(x)=L_{n}^{(\alpha)}\left(x^{2}\right)
$$

with $\alpha$ arbitrary.

$$
f_{2 n+1}(x)=x L_{n}^{(\alpha+1)}\left(x^{2}\right)
$$

3. Remarks and generalization. It may be of interest to examine the more general problem of solving the functional equation

$$
\sum \gamma_{n} f_{n}(x) g_{n}(x) t^{n}=f(t) e^{x^{k} a(t)+y^{k} b(t)} g\{x y c(t)\},
$$

where $f_{n}(x), g_{n}(x)$ are polynomials of exact degree $n$ and highest coefficient equal to 1 . Hence we require

$$
a_{0}=a_{1}=\ldots=a_{k-1}=0, \quad b_{0}=b_{1}=\ldots=b_{k-1}=0 .
$$

If $a_{k}=0$ we obtain from (3.1)

$$
\sum \gamma_{n} f_{n}(x) t^{n}=g\left\{x c_{1} t\right\}
$$

which leads to

$$
\sum \gamma_{n} t^{n}=g\left(c_{1} t\right)
$$


Hence

$$
\sum \gamma_{n} x^{n} t^{n}=\sum \gamma_{n} f_{n}(x) t^{n}
$$

We thus conclude that, if $a_{k}=0$, then

$$
f_{n}=x^{n}
$$

Similar remarks apply in case $b_{k}=0$. Since this solution is trivial, we assume that

$$
a_{k}=b_{k}=1 \text {. }
$$

With (3.2) in mind we get from formula (3.1)

$$
\sum \gamma_{n} f_{n}(x) t^{n}=e^{t^{k}} g\left(x t c_{1}\right)
$$

and

$$
\sum \gamma_{n} g_{n}(y) t^{n}=e^{t^{k}} g\left(y t c_{1}\right)
$$

which clearly shows that

$$
f_{n}(x)=g_{n}(x) .
$$

Hence the problem is reduced to the previous problem which was treated in $\S 2$.

\section{REFERENCE}

1. G. Szegó, Orthogonal polynomials, American Mathematical Society Colloquium Publications. vol. 23, Revised edition, New York, 1959.

Texas Technological College

LUBBOCK

TEXAS, U.S.A.
DUKE UNIVERSITY

DURHAM

N. Carolina, U.S.A. 